\title{
Does Consumer Behaviour on Meat Consumption Increase Obesity? - Empirical Evidence from European Countries
}

\author{
Hasan Mohammad Monirul \\ Department of Economic and Technological Change, Center for Development Research (ZEF), University of Bonn, Germany \\ Email address: \\ monir1021@gmail.com
}

To cite this article:

Hasan Mohammad Monirul. Does Consumer Behaviour on Meat Consumption Increase Obesity? - Empirical Evidence from European Countries. International Journal of Economic Behavior and Organization. Vol. 2, No. 1, 2014, pp. 1-5. doi: 10.11648/j.ijebo.20140201.11

\begin{abstract}
The study shows the association between consumer behavior on meat consumption and obesity of individuals in the European countries. Consumer behavior in terms of meat consumption and life-style has significant impact on obesity which is defined by Body Mass Index (BMI) calculated as kilogram per meter square. The primary sample survey which was conducted online, received the voluntary response from European nationals. The study finds that increasing proportion of meat in the meal has significant positive association in increasing the BMI. The study shows that age and meat consumption have significant positive association with increasing BMI, however, education, income and hours of physical exercise have negative association with increasing BMI. In order to analyze the complex and causal relationship among the variables, an econometric regression technique has been used. As the dependent variable BMI is an ordered categorical variable, ordered logistic model is used to analyze the model.
\end{abstract}

Keywords: Obesity, Meat Consumption, Gender, European Consumers, Consumer Behavior

\section{Introduction}

The living way of modern people changes continuously where people work more, return back home exhausted without having the mood to prepare an appropriate meal, consume pre-cooked or processed food and most of them in general do not include exercise in their daily schedule. Nowadays people do not have free time to prepare better and healthy food and when they have they spent it on internet or watching TV. The lack of proper understanding of healthy food and highly dependence on processed food can make an individual obese. Besides the preference for the meat consumption has increase in the whole world because of the increasing income. Existing study finds that Meat free diet is associated with low prevalence of obesity (Key and Davey, 1996). According to a survey conducted by Wang and Beydoun (2009) regarding the relationship between obesity and meat consumption, the results showed that high consumption of meat and meat products (like sausages) are associated with high Body Mass Index (BMI) for the US citizen in cross sectional studies. They found that individual consuming higher amount of meat (upper quintile) was nearly $27 \%$ more likely to be obese, compared to lower amount consumers. Higher intake of meat or meat products is associated with higher BMI whereas intake of vegetable and fruits may lower BMI. A positive correlation also exists between income and BMI and in addition obesity rates are increasing as per capita incomes are increasing as well (Seidell and Rissanen, 1998; WHO, 2003; Swinburn et al., 2004).

Regarding general obesity, Bendsen et al. (2013) found that in most observational studies there is an inverse association or no association between beer intake and body weight for women whereas a positive association or no association in men. Nonetheless, a diet low in fruits and red meat but high in snack food were associated with higher waist circumferences for both male and female; however for female less vegetable, more butter and high-fat dairy product, more poultry and processed meat are likely the determinants of the gain at the waist (Halkjaer et al., 2009).

Kimokoti et al. (2012) find that demographic and lifestyle may modify the association between dietary patterns and the metabolic syndrome. However, though men and women had similar food intake patterns, women are less positively associated with higher BMI than men (Togo et al., 2001). Their study suggests using of other techniques than diet index, factor analysis or clustering 
analysis. In a study regarding the robustness of the methodology, Togo et al. (2004) found no significant association between factor scores and subsequent BMI changes for the Danish population using factor analysis technique. On the other hand Wang and Beydoun (2009) applied linear and logistic regression analysis to find the potential confounders of higher BMI. Soup consumption is not associated with metabolic syndrome however; there is an inverse relationship between soup consumption and body weight status in US adults (Zhu and Hollis, 2013).

Xiao et al. (2013) used education and income as the indicator of socio-economics status in response to BMI. Study finds that less education of the women is positively associated with higher BMI whereas higher income of men is positively associated with higher BMI in Zhejiang province of China. According to World Health Organization (WHO), "Worldwide obesity has more than doubled since 1980, in 2008, more than 1.4 billion adults, 20 and older, were overweight. Of these more than 200 million men and about 300 million women were obese and $65 \%$ of the world's population live in countries where overweight and obesity kills more people than underweight". So obesity could be defined as "an abnormal or excessive fat accumulation that may impair health". The tool that helps us to determine if a person is obese or not is BMI which is calculated by dividing the mass of an individual by the square of his/her height (BMI=Masse $(\mathrm{Kg}) /$ Height $\left(\mathrm{m}^{2}\right)$.

The purpose of this study is to examine how much BMI is associated with some personal characteristics and food habits like age, education, gender, meat consumption, taking yogurt, income, hours of physical exercise, hours of watching television etc. The research question can be specifically written as- Does consumer behavior on meat consumption increase obesity?

\section{Data and Methods}

\subsection{Categorizing the Dependent Variable- BMI}

The dependent variable BMI is measured by taking the height and weight of an individual. As BMI is always analyzed in ranges by the nutritionist because of health issues, the study categorizes it according to the Sizer and Whitney (1994) nutrition measurement concept of BMI classification. It is very important to mention that the BMI ranges can be varied according to gender. Female have different classification than male. This classification of the BMI incorporate the gender based BMI because the unified BMI classification for men and women mislead the information.

Table 1. Body Mass Index (BMI)

\begin{tabular}{llll}
\hline Men & Women & Risk Factor & BMI class \\
\hline$<20.7$ & $<19.1$ & Underweight & 1 \\
20.7 to 26.4 & 19.1 to 25.8 & Normal & 2 \\
26.4 to 27.8 & 25.8 to 27.3 & Marginally overweight & 3 \\
27.8 to 31.1 & 27.3 to 32.2 & Overweight & 4 \\
$>31.1$ & $>32.3$ & Severe overweight & 5 \\
\hline
\end{tabular}

Source: Sizer and Whitney, 1994

According to Table 1, less than 20.7 is the underweight which is the BMI class 1 and more than 32.3 is the severe overweight which is BMI class 5.

\subsection{Analytical Framework}

The analytical framework comprises the regression framework and the rationale for using the regression model. The rationale behind the uses of ordered logistic model is to find out the severity of the risk factors which are associated with BMI. BMI as a continuous variable may give us the extent of change in the dependent variable due to change in the explanatory variable but that would be very minutest amount and it would be very difficult to measure the risk factors associated with BMI. So BMI is classified into categories because of the simplicity and to keep pace with the existing nutrition research. As the dependent variable is naturally ordered hence it is required to use ordered logistic model to find out the determinants of the higher BMI. The beauty of logistic model is that it gives the probability if approaching a higher risk factor level in comparison to other risk factor levels due to change in each explanatory variable.

The ordered logistic model can be described as equation (1),
(2) and (3). According to Cameron and Trivedi (2005), the starting point is an index model, with single latent variable

$$
y_{i}^{*}=x^{\prime} \beta_{i}+u_{i} \text { where } u_{i} \sim N I D(0,1)
$$

In the equation (1), $x$ doesn't include intercept. As $y^{*}$ crosses a series of increasing unknown thresholds we move up the ordering of alternatives. As for example, for a very low $y^{*}$ health status is poor, for $y^{*}>\alpha 1$ health status improves to fair, for $y^{*}>\alpha 2$ it improves further to good, and so on.

In general for an $m$-alternative ordered model we define

$$
y_{i}=\mathrm{j} \text { if } \alpha_{j-1}<y^{*} \leq \alpha_{j}
$$

Where $\alpha_{0}=-\infty$ and $\alpha_{m}=\infty$. Then

$$
\begin{gathered}
\operatorname{Pr}\left[y_{i}=j\right]=\operatorname{Pr}\left[\alpha_{j-1}<y_{i}^{*} \leq \alpha_{j}\right] \\
=\operatorname{Pr}\left[\alpha_{j-1}<x_{i}^{\prime} \beta+u_{i} \leq \alpha_{j}\right] \\
=\operatorname{Pr}\left[\alpha_{j-1}-x_{i}^{\prime} \beta<u_{i} \leq \alpha_{j}-x_{i}^{\prime} \beta\right. \\
=F\left(\alpha_{j}-x_{i}^{\prime} \beta\right)-F\left(\alpha_{j-1}-x_{i}^{\prime} \beta\right),
\end{gathered}
$$


Where, $F$ is the Cumulative Density Function (cdf) of $u_{i}$. The regression parameters $\beta$ and the $(\mathrm{m}-1)$ threshold parameters $\alpha_{1}, \ldots \alpha_{m-1}$ are obtained by maximizing the $\log$ likelihood function

$$
L=\ln L_{N}=\sum_{i=1}^{N} \sum_{j=1}^{M} y_{i j} \ln p_{i j}
$$

Where $p_{i j}=F_{j}\left(x_{i}, \beta\right)$ is a function of parameters $\beta$ and regressors.

If we maximize the log likelihood function of (4) with respect to $p_{i j}$ defined in (3) we will obtain the parameters $\alpha_{1} \ldots \ldots \alpha_{m-1}$.

For the ordered logit model $u$ is logistic distributed with $F(z)=\frac{e^{z}}{1+e^{z}}$

The sign of the regression parameters $\beta$ can be immediately interpreted as determining whether or not the latent variable $y^{*}$ increases with the regressors. For marginal effect in the probabilities

$$
\frac{\partial \operatorname{Pr}\left[y_{i}=j\right]}{\partial x_{i}}=\left\{F^{\prime}\left(\alpha_{j-i}-x_{i}^{\prime} \beta\right)-F^{\prime}\left(\alpha_{j}-x_{i}^{\prime} \beta\right)\right\} \beta
$$

Where $F^{\prime}$ denotes the derivative of $F$.

\subsection{Data}

A primary sample survey was conducted for the outreach of around 1000 persons on January 2012 in the European countries. The questionnaire was designed to conduct a survey in online which was publicly accessed and taking part in this survey was voluntary. So the mode of collecting data was by using written questionnaire. It is also mentioned that the identity of the interviewed persons were anonymous. The age range of the interviewed persons was between 16 and 45 . A total of 135 observations had been collected of which $40 \%$ were male and $60 \%$ female. The data set is covering the information of $30 \%$ Greek, $20 \%$ German, $8 \%$ Hungarian, $8 \%$ Italian and the rest of the information from other European countries. The online written survey comprises questions related to meat consumption, education, hours of physical exercise, way of living, occupation, height, weight, monthly income, consumption of yogurt and watching television etc.

\subsection{Descriptive Statistics}

Selected variables to run the regression model are: age in years, gender, education level, meat consumption as a percentage that occupies in a regular meal, frequency of weekly yogurt consumption, monthly income in Euros, hours dedicated to physical exercise per week and hours dedicated to watch TV per day. The descriptive statistics of these variables are displayed in Table 2. Regarding education level, 43\% got Bachelor degree, 41\% Master degree, $2 \%$ Doctoral studies, while 4\% Post-doctoral studies. Finally, $10 \%$ is still attending to the High School. The mean age of the respondents are 25 age and the maximum age is 45 . Respondent eats meat more than a quarter of their total meal each time where the maximum meat consumption is three-quarter per meal. The frequency of yogurt consumption is almost 6 times per week. The mean income of the respondent is 578 euro where the maximum amount is 3000 euro per month. An average of 4 hours of physical exercise and 1 hour of television watching were reported by the respondent.

Table 2. Descriptive statistics

\begin{tabular}{lllll}
\hline Variable & Mean & SD & Min & Max \\
\hline Age in years & 25.11 & 4.91 & 16 & 45 \\
Meat consumption (ratio of full meal) & 0.32 & 0.13 & 0.25 & 0.75 \\
Number of yogurt consumption per week & 5.80 & 15.51 & 0 & 7 \\
Income per month in Euros & 578 & 481.80 & 0 & 3000 \\
Hours of physical exercise per week & 4.02 & 2.79 & 1 & 15 \\
Hours watching TV per day & 1.15 & 1.35 & 0 & 8 \\
\hline
\end{tabular}

Source: Author's calculation

\subsection{Regression model}

The linear regression model cannot be appropriate here as the dependent variable is ordered in nature. So is it required to use non-linear model. The regression model which is explained in the analytical framework (section 2.2) can be described in a very simple form as

$$
B M I_{i}^{*}=X_{i}^{\prime} \beta+\delta D_{i}+\varepsilon_{\boldsymbol{i}}
$$

Where,

$B M I_{i}^{*}=$ BMI class for individual $i$ (ordered from 1 to 5 )

$\mathrm{X}_{\mathrm{i}}=$ vector of observed continuous variables like Age, meat consumption, yogurt consumption, income, hours of physical exercise, hours of watching television etc. for individual $i$.

$\mathrm{D}_{\mathrm{i}}=$ Dummy of gender and educational strata like bachelor, master, $\mathrm{PhD}$ etc.

\section{Result}

Ordered Logistic Regression Model is used to analyze the determinants of BMI increase. In Table 3 the coefficients of the model and the odds ratios are displayed. The regression with the coefficients offers a first insight of the sign and significance of the variables, while the results containing the odds ratios provide with information about the quantitative 
impact of the exogenous variables on the BMI.

Table 3. Ordered Logistic Regression Model (dependent variable- BMI class)

\begin{tabular}{|c|c|c|c|c|}
\hline Variables & coefficient & SE & odds ratio & SE \\
\hline Age of respondent & $0.214 * * *$ & 0.054 & $1.2 * * *$ & 0.067 \\
\hline Female & $-1.235^{* * *}$ & 0.456 & $0.29 * * *$ & 0.133 \\
\hline meat consumption (ratio of full meal) & $2.592 *$ & 1.479 & $13.35^{*}$ & 19.76 \\
\hline Education: Bachelor & $-1.321 * *$ & 0.646 & $0.26 * *$ & 0.172 \\
\hline Masters & $-2.075 * * *$ & 0.656 & $0.126^{* *}$ & 0.084 \\
\hline $\mathrm{PhD}$ & -1.950 & 1.474 & 0.142 & 0.210 \\
\hline M. Phil & -2.981 & 1.998 & 0.051 & 0.101 \\
\hline Post-doctorate & $-3.817 * * *$ & 1.380 & $0.022 * * *$ & 0.030 \\
\hline Frequency of yogurt consumption /week & 0.006 & 0.013 & 1.006 & 0.013 \\
\hline Monthly income (in Euro) & -0.000 & 0.000 & 1.000 & 0.000 \\
\hline Hours for physical exercise in a week & -0.093 & 0.073 & 0.911 & 0.067 \\
\hline Hours of watching television every day & 0.082 & 0.152 & 1.086 & 0.165 \\
\hline
\end{tabular}

Note: $* * * \mathrm{p}<0.01, * * \mathrm{p}<0.05, * \mathrm{p}<0.1$

Source: Author's calculation

\subsection{Discussion of Results}

As displayed in Table 3, the age of the respondent and the meat consumption variable have a positive and significant impact on the BMI, while the gender (being a female) and the education level have a negative and significant impact on the BMI. In the case of the education level, a significant impact is observed in almost all categories (especially for the category of Bachelor with a significant level of $5 \%$, Master 1\% level and Post Doctorate at $1 \%$ level). For Master in Philosophy, it is insignificant because of too small observations. Other variables, such as yogurt consumption, income, hours of physical exercise and hours watching TV have no significant impact on the BMI but the sign of these variables shows the expected relationship with BMI. To analyze the result it can be said that for one unit increase in age, one can expect a 0.21 increase in the log odds of being in a higher level of BMI, given that all other variables in the model are held constant. For being a female respondent one can expect a 1.23 decrease in the log odds of being in a higher level of BMI, given that all other variables in the model are held constant. For the main variable - meat consumption, it can be said that for one unit increase (from quarter to half or half to quarter of the share of full meal), one can expect a 2.6 increase in the log odds of being in a higher level of BMI, given all other variables in the model are held constant. As the model is ordered logit model, it is required to find out the odds ratios of the variables. In case of meat consumption, for one unit increase in meat consumption, the odds of the low and middle categories of BMI vs high categories of BMI are 1.2 times greater, given that all other variables are held constant. Because of the proportional odds assumption, the same increase 1.2 times found between low vs middle and high category of BMI. From table 3 it is clear that if age increases, the BMI also increases. If an individual increases his/her meat consumption from one quarter to half of the total meal, the probability of BMI increase statistically significantly increases. Female are less responsive to the increasing BMI in the model. It is found that the level of education reduces the level of BMI significantly.

In discussing the results it is worth mentioning the distinction between the earlier literature and the presented results in this paper. Unlike the earlier studies, this paper incorporated age of individual, physical exercise, consumption of yogurt and hours of television watching as control variables to validate the results of the causation of meat consumption to higher BMI. The establishment of causality is verified with the incorporation of income variable and education variables. It is found in the presented result that the incorporation of new variables such as income and other individual characteristics don't change the sign and significance of the main explanatory variable (meat consumption) to the outcome variable BMI. Like Wang and Beydoun (2009) this paper also finds the association with meat consumption with BMI, but here it controls results with income variable. Unlike Seidell and Rissanen (1998) and Swinburn et al. (2004), this paper finds no statistically significant association between income and BMI increase. The paper also finds that women are less likely to have lower BMI than men by using econometric analysis which is similar type of result from Togo et al. (2001) using factor analysis. The paper consistently and significantly supports the impact of higher education on lowering BMI. Higher education which means higher level of activities and more consciousness of proper food and health helps the probability of having lower BMI than the low educated people. Xiao et al. (2013) finds similar results only for low educated women in rural China but this paper finds this association for both men and women in the European countries. An increase in the hours of physical exercise doesn't produce any significant correlation with increasing BMI except the expected negative sign. The same is true for television watching.

\section{Conclusion}

The increase in age of the respondent and the amount of meat consumption has a positive and significant impact on the increasing BMI whereas being female and higher 
education level have a negative and significant impact on BMI. As gender is a binary variable, it means that women tend to have less obesity problems than men. One plausible explanation might be women are more conscious about their appearance. In addition, more educated people are less prone to have obesity problems probably due to more and better access to health and diet information. Although having expected sign the other variables that did not show any significant impact (for instance: yogurt consumption, income, hours of physical exercise) may be because of the small data set. If we could increase the sample size, it would be possible to find a significant result on BMI. Hence it is clear from the study that increasing the proportion of meat in the daily meal will increase obesity. The contribution of this paper to the existing literature is that it also deals with age, income and other personal characteristics as control variables to validate the results. Different levels of education, age, gender and meat consumption variables have significantly explained the changes in the depended variable BMI class. Therefore, higher proportion of meat in the meal contributes to higher level of BMI.

\section{References}

[1] Bendsen NT, Christensen R, Bartels EM, Kok FJ, Sierksma A, Raben A, Astrup A (2013). Is beer consumption related to measures of abdominal and general obesity?-A systematic review and meta-analysis. Nutrition Reviews, 71: 67-87.

[2] Cameron AC, Trivedi PK (2005). MicroeconometricsMethods and applications. Cambridge University Press, 1: 518-519.

[3] Halkjaer J, Tjønneland A, Overvad K, Sørensen TI (2009). Dietary Predictors of 5-Year Changes in Waist Circumference. J. Am. Diet. Assoc., 109: 1356-1366.

[4] Key T, Davey G (1996). Prevalence of obesity is low in people who do not eat meat. BMJ, 313: 816-817.
[5] Kimokoti RW, Gona P, Zhu L, Newby PK, Millen BE, Brown LS, D_Agostino RB, Fung TT (2012). Dietary Patterns of Women Are Associated with Incident Abdominal Obesity but Not Metabolic Syndrome. J. Nutr., 142: 1720-1727.

[6] Seidell JC, Rissanen AM (1998). Time Trends in the Worldwide Prevalence of Obesity. In Bray GA, Bouchard C, James WPT (eds). Handbook of Obesity. New York, NY: Marcel Dekker.

[7] Sizer FS, Whitney EN (1994). Hamilton and Whitney's Nutrition Concepts and Controversies, 6th ed. St Paul, Minn, West Publishing.

[8] Swinburn B, Caterson I, Seidell JC, James WPT (2004). Diet, Nutrition and the Prevention of Excess Weight Gain and Obesity. Public Health Nutrition, 7: 123-146.

[9] Togo P, Osler M, Sørensen TI, Heitmann BL (2001). Food intake patterns and body mass index in observational studies. Int. J. Obes. Relat. Metab. Disor., 25: 1741-1751.

[10] Togo P, Osler M, Sørensen TI, Heitmann BL (2004). A longitudinal study of food intake patterns and obesity in adult Danish men and women. Int. J. Obes. Relat. Metab. Disor., 28: 583-93.

[11] Wang Y, Beydoun MA (2009). Meat consumption is associated with obesity and central obesity among US adults. Int. J. Obes., 33: 621-628.

[12] WHO: World Health Organization (2003). Diet, Nutrition, and the Prevention of Chronic Diseases. WHO Technical Report Series 916. Geneva.

[13] Xiao Y, Zhao N, Wang H, Zhang J, He Q, Su D, Zhao M, Wang L, Zhang X, Hu R et al. (2013). Association between socioeconomic status and obesity in a Chinese adult population. BMC Public Health, 13: 355.

[14] Zhu Y, Hollis JH (2013). Soup Consumption Is Associated with a Reduced Risk of Overweight and Obesity but Not Metabolic Syndrome in US Adults: NHANES 2003-2006. PLoS ONE, 8: e75630. 198. 